\title{
Analysis of RC Interconnections Under Ramp Input
}

\author{
ANDREW B. KAHNG and SUDHAKAR MUDDU \\ University of California, Los Angeles
}

\begin{abstract}
We give new methods for calculating the time-domain response for a finite-length distributed $R C$ line that is stimulated by a ramp input. The following are our contributions. First, we obtain the solution of the diffusion equation for a semiinfinite distributed $R C$ line with ramp input. We then present a general and, in the limit, exact approach to compute the time-domain response for finite-length $R C$ lines under ramp input by summing distinct diffusions starting at either end of the line. Next, we obtain analytical expressions for the finite time-domain voltage response for an open-ended finite $R C$ line and for a finite $R C$ line with capacitive load. The delay estimates using this method are very close to SPICE-computed delays. Finally, we present a general recursive equation for computing the higher-order diffusion components due to reflections at the source and load ends. Future work extends our method to response computations in general interconnection trees by modeling both reflection and transmission coefficients at discontinuities.
\end{abstract}

Categories and Subject Descriptors: B.7.1 [Integrated Circuits]: Types and Design StylesVLSI; B.7.2 [Integrated Circuits]: Design Aids-layout; F.2.1 [Analysis of Algorithms and Problem Complexity]: Numerical Algorithms and Problems-computation of transforms

General Terms: Algorithms, Design, Performance, Theory, Verification

Additional Key Words and Phrases: Diffusion equation analysis, ramp input response, VLSI interconnects

\section{INTRODUCTION}

Estimating delays on VLSI interconnections is a key element in timing verification, gate-level simulation and performance-driven layout design. Because of the highly resistive nature of the wires, present-day tools model the wires inside integrated circuits as distributed $R C$ lines. The analysis of finite $R C$ transmission lines with step input is widely discussed in the

This work was supported by NSF grant MIP-9257982 and a gift from Cadence Design Systems, Inc.

A preliminary version of this work appeared in Proceedings of ACM/IEEE Design Automation Conference, June 1996.

Authors' address: Computer Science Department, University of California, Los Angeles, Los Angeles, CA 90095-1596.

Permission to make digital/hard copy of part or all of this work for personal or classroom use is granted without fee provided that the copies are not made or distributed for profit or commercial advantage, the copyright notice, the title of the publication, and its date appear, and notice is given that copying is by permission of the ACM, Inc. To copy otherwise, to republish, to post on servers, or to redistribute to lists, requires prior specific permission and/or a fee.

(C) 1997 ACM 1084-4309/97/0400-0168 $\$ 03.50$ 
literature, e.g., [Antinone and Brown 1983; Peirson and Bertnolli 1969; Wilnai 1971; Sakurai 1983]. The standard approach is to first calculate the transfer function; then, by approximating the transfer function, both transform-domain and time-domain responses are obtained for different configurations of the finite $R C$ line with step input. Using different approaches to invert the Laplace transform of the response, Kahng and Muddu [1994, 1996], Mattes [1993], and Rao [1995] have all obtained the exact timedomain response for a finite-length open-ended $R C$ line. The most recent of these works, by Rao, also extends the traditional transform-domain analysis to calculate the time-domain response for a finite $R C$ line with capacitive load impedance. A direct solution of the open-ended finite $R C$ line response, i.e., directly in the time-domain as an infinite series, was first given by Kaufman and Garrett [1962]. Kahng and Muddu [1994, 1996] calculated the time-domain response in a finite distributed $R C$ line with source and load impedances; the total response was shown to be equal to an infinite sum of diffusion equation solutions, with each diffusion starting at either the source or load end of the line.

None of these previous works gives an understanding of the interconnect response to input signals with nonzero transition time. It is more reasonable to model the input signal from drivers to interconnect lines as a finite ramp input. However, analysis of finite $R C$ transmission lines under ramp input has received comparatively little attention. Kaupp [1989] analyzed $R C$ interconnections under finite ramp input by assuming infinitely long transmission lines. Extending this work, Abuelma'atti [1990] approximated the transfer function of a semiinfinite line using a linear function, and proposed a model for $R C$ lines under ramp input. Recently, Menezes et al. [1994] proposed a methodology for $R C$ interconnect synthesis under ramp input using the first few moments of the transfer function. Nevertheless, no analytical solution for the time-domain transient response of a finite distributed $R C$ line with source and load impedances under finite ramp input has been obtained in the literature.

We analyze finite distributed $R C$ lines under ramp input, using a new technique based on solving the diffusion equation and applying the method of images [Kahng and Muddu 1994, 1995]. Using this new technique, we are able to analytically obtain the transient time-domain response of a finite $R C$ line for different cases of source and load impedances. Our contributions are the following:

-We first obtain the time-domain response of a semi-infinite $R C$ line for both infinite and finite ramp inputs by solving the diffusion equation with appropriate boundary conditions. This result matches the semiinfinite ramp response given in Kaupp [1989].

-We then provide a general approach to compute the time-domain response for finite $R C$ lines by summing distinct diffusions starting at either end of the line; each of these can be viewed as traveling either forward or backward along the line, in analogy with reflections. In the 


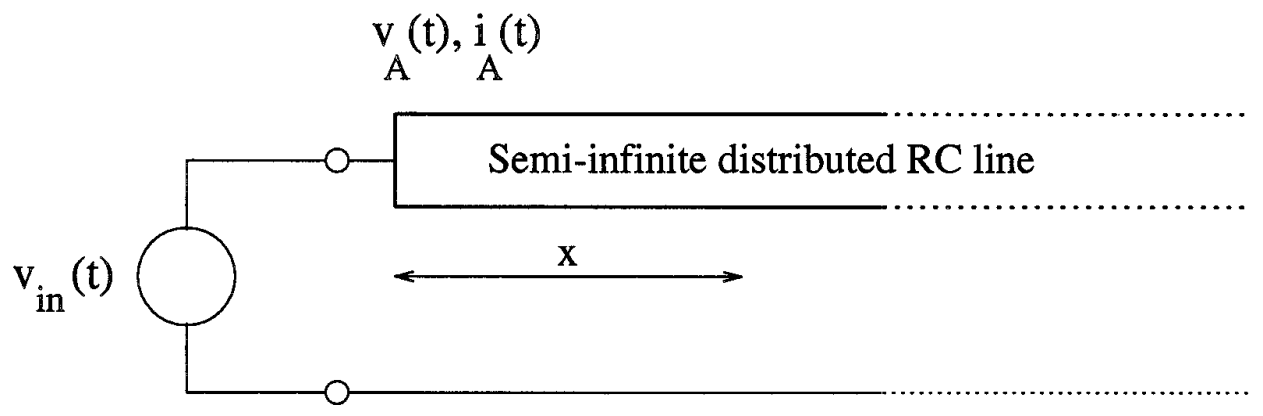

Fig. 1. A semi-infinite distributed $R C$ line and position $x$ along the line.

limit, this approach gives an exact analysis, but only a few reflections are needed to achieve accurate response computations.

-We obtain the analytical expressions for the time-domain voltage response under ramp input for an open-ended finite $R C$ line and for a finite $R C$ line with capacitive load. To the best of our knowledge, these results are completely new; there is no previous literature on this subject. We compare delay estimates from our approach and from SPICE with URC (Uniform Distributed $R C$ ) model for $R C$ lines: using only the first few reflected diffusion components in the voltage response, our delay estimates are very close to SPICE-computed delays. Finally, we present a general recursive equation for computing the higher order diffusion components due to reflections at the source and load ends of the interconnect line. Our method is simple and can be extended to response computations in general interconnection trees by modeling both reflection and transmission coefficients at discontinuities.

\section{SEMI-INFINITE $R C$ LINE ANALYSIS}

Consider the semi-infinite distributed $R C$ line shown in Figure 1. The voltage and current on a uniform distributed $R C$ line is governed by the diffusion equation

$$
r c \frac{\partial v(x, t)}{\partial t}=\frac{\partial^{2} v(x, t)}{\partial x^{2}}
$$

where $v(x, t)$ is the voltage on the line at position $x$ and at time $t$, and $r$ and $c$ are resistance and capacitance per unit length, respectively. The solution to the diffusion equation under various boundary conditions has been well studied [Kevorkian 1990]. The work of Kahng and Muddu [1994] showed that the time-domain response of a finite-length $R C$ line with step input was equal to an infinite sum of diffusion equation solutions, with each diffusion starting at either the source or the load end of the line.

Here, we are interested in calculating the response for a finite $R C$ line under finite ramp input. For a semi-infinite $R C$ line the total voltage on the line is due to only the incident diffusion component, i.e., the incident 

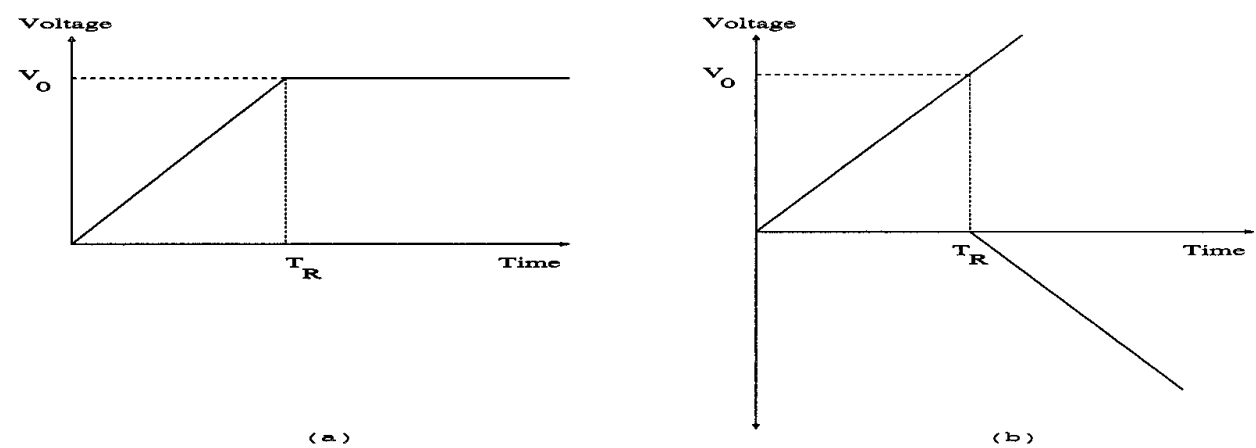

Fig. 2. A ramp input function: (a) finite ramp with rise time $T_{R}$; and (b) finite ramp decomposed into two shifted infinite ramps.

propagation of voltage. We will first solve the above diffusion equation for the semi-infinite line, then address the more general case where the total voltage response on the line is the sum of incident and reflected diffusion components. The initial and boundary conditions for the semi-infinite line under finite ramp input (Figure 2) are

$$
\mathrm{IC}: v(x, 0)=0
$$

for all $x \geq 0$

$\mathrm{BC} 1: v(0, t)=v_{i n}(t)=\frac{V_{0}}{T_{R}}\left[t U(t)-\left(t-T_{R}\right) U\left(t-T_{R}\right)\right] \quad$ for all $\quad t \geq 0$

where $U(t)$ denotes the step function.

We first consider an infinite ramp input since any finite ramp can be expressed as the sum of two shifted infinite ramps (Figure 2); the timedomain response for a shifted infinite ramp can be derived from the infinite ramp response by a change of time variable and the response for a finite ramp is obtained by adding the responses due to two infinite ramps. Using $u(x, t)$ to represent the response for an infinite ramp input, the diffusion equation and new boundary conditions are: ${ }^{1}$

$$
\begin{array}{crl}
r c \frac{\partial u(x, t)}{\partial t} & =\frac{\partial^{2} u(x, t)}{\partial x^{2}} & \\
\text { IC: } u(x, 0) & =0 & \text { for all } x \geq 0 \\
\text { BC1: } u(0, t) & =\frac{V_{0}}{T_{R}} \cdot t \cdot U(t) & \text { for all } t \geq 0
\end{array}
$$

\footnotetext{
${ }^{1}$ In the transform and time domains, we use $U(x, s)$ and $u(x, t)$ to, respectively, indicate the response for the infinite ramp input, and $V(x, s)$ and $v(x, t)$ to, respectively, indicate the response for the finite ramp input.
} 
The diffusion equation for step input has a boundary condition that is constant with respect to time. For a ramp input this boundary condition is a function of time, so it is difficult to derive the solution in the same way as for a step input. However, differentiating the diffusion equation in time and using the variable $w(x, t)=\partial u(x, t) / \partial t$, we again obtain a diffusion equation,

$$
r c \frac{\partial w(x, t)}{\partial t}=\frac{\partial^{2} w(x, t)}{\partial x^{2}}
$$

with initial and boundary conditions obtained by taking the time derivative of the boundary conditions of $u(x, t)$. The initial condition remains the same, but the boundary condition for the new diffusion equation becomes constant in time, i.e., similar to that for a step input:

$$
\begin{aligned}
& \text { IC: } w(x, 0)=0 \quad \text { for all } \quad x \geq 0 \\
& \mathrm{BC} 1: w(0, t)=\frac{V_{0}}{T_{R}} U(t) \quad \text { for all } \quad t \geq 0
\end{aligned}
$$

The solution for the diffusion equation under step input can be obtained using the parabolic substitution of the variable $\eta=x \sqrt{r c /(2 t)}$ [Kahng and Muddu 1994] as

$$
w(\eta)=C_{1} \int_{0}^{\eta} e^{\left(-y^{2} / 2\right)} d y+C_{2}
$$

The initial condition (IC) that the line is quiet at $t=0$ implies $C_{1}=-\sqrt{2 /(\pi)} C_{2}$. The boundary condition BC1 implies that the derivative of voltage with respect to time at the front end of the line (i.e., at position $x=0$ ) is constant and equal to $V_{0} / T_{R}$ for all $t>0$. Using this condition in the above equation yields $C_{2}=\left(V_{0} / T_{R}\right)$. Therefore,

$$
w(x, t)=\frac{V_{0}}{T_{R}}\left[1-\operatorname{erf}\left(\frac{\eta}{\sqrt{2}}\right)\right]=\frac{V_{0}}{T_{R}} \operatorname{erfc}\left(\frac{b}{\sqrt{4 t}}\right),
$$

where $x$ is the position at which the response is calculated and $b=x \sqrt{r c}=$ $\sqrt{R_{x} C_{x}}, R_{x}=x r, C_{x}=x c$. From this, the incident diffusion component for the semi-infinite $R C$ line under infinite ramp input, which we denote as $u_{I}(x, t)$, can be derived as: ${ }^{2}$

\footnotetext{
${ }^{2}$ Observe that Eq. (3) also applies for the finite-length distributed $R C$ line with perfectly matched load because there is no reflection at the load, i.e., there is only the incident diffusion voltage along the line.
} 


$$
\begin{aligned}
u_{I}(x, t) & =\int_{\tau=0}^{\tau=t} w(x, \tau) d \tau \\
& =\int_{\tau=0}^{\tau=t} \frac{V_{0}}{T_{R}} \operatorname{erfc}\left(x \sqrt{\frac{r c}{4 \tau}}\right) d \tau \\
& =\frac{V_{0}}{T_{R}}\left[\left(t+\frac{R_{x} C_{x}}{2}\right) \operatorname{erfc}\left(\sqrt{\frac{R_{x} C_{x}}{4 t}}\right)-\sqrt{\frac{R_{x} C_{x} t}{\pi}} e^{-\left(R_{x} C_{x} /(4 t)\right)}\right] U(t) \\
& =\frac{V_{0}}{T_{R}}\left[\left(t+\frac{b^{2}}{2}\right) \operatorname{erfc}\left(\frac{b}{\sqrt{4 t}}\right)-b \sqrt{\frac{t}{\pi}} e^{-b^{2} /(4 t)}\right] U(t) .
\end{aligned}
$$

Then, the time-domain response for the incident diffusion component $v_{I}(x, t)$ with a finite ramp input can be written in terms of the infinite ramp response: ${ }^{3}$

$$
\begin{aligned}
v_{I}(x, t)= & u_{I}(x, t)-u_{I}\left(x, t-T_{R}\right) \\
= & \frac{V_{0}}{T_{R}}\left[\left(t+\frac{b^{2}}{2}\right) \operatorname{erfc}\left(\frac{b}{\sqrt{4 t}}\right)-b \sqrt{\frac{t}{\pi}} e^{-b^{2} /(4 t)}\right] U(t) \\
& -\frac{V_{0}}{T_{R}}\left[\left(t-T_{R}+\frac{b^{2}}{2}\right) \operatorname{erfc}\left(\frac{b}{\sqrt{4\left(t-T_{R}\right)}}\right)\right. \\
& \left.+b \sqrt{\frac{\left(t-T_{R}\right)}{\pi}} e^{-b^{2} /\left(4\left(t-T_{R}\right)\right)}\right] U\left(t-T_{R}\right)
\end{aligned}
$$

As expected, the second term in the above equation is zero for $t \leq T_{R}$, so the finite ramp response is given by Eq. (3) for $t \leq T_{R}$ and by Eq. (4) for $t \geq T_{R}$.

The infinite ramp response in the transform domain can be obtained by taking the Laplace transform of the time-domain response given in Eq. (3), which yields $U_{I}(x, s)=\left(V_{0} / T_{R} s^{2}\right) \cdot e^{-\sqrt{R_{x} C_{x}}}$. Similarly, the finite ramp

\footnotetext{
${ }^{3}$ We can also compute the response for ramp input by using the transfer function of the semi-infinite $R C$ line, $H_{I}(x, s)=e^{-x \sqrt{r c s}}$ and the infinite ramp input, $U_{i n}(s)=V_{0} / T_{R} \cdot 1 / s^{2}$. The incident diffusion component in the transform domain is

$$
U_{I}(x, s)=\frac{V_{0}}{T_{R} s^{2}} \cdot e^{-\sqrt{R_{x} C_{x} s}}=\frac{V_{0}}{T_{R} s^{2}} \cdot e^{-b \sqrt{s}} .
$$

The time-domain response obtained by applying the inverse Laplace transform tables in Campbell and Foster [1957] is exactly equal to the response computed in Eq. (3).
} 


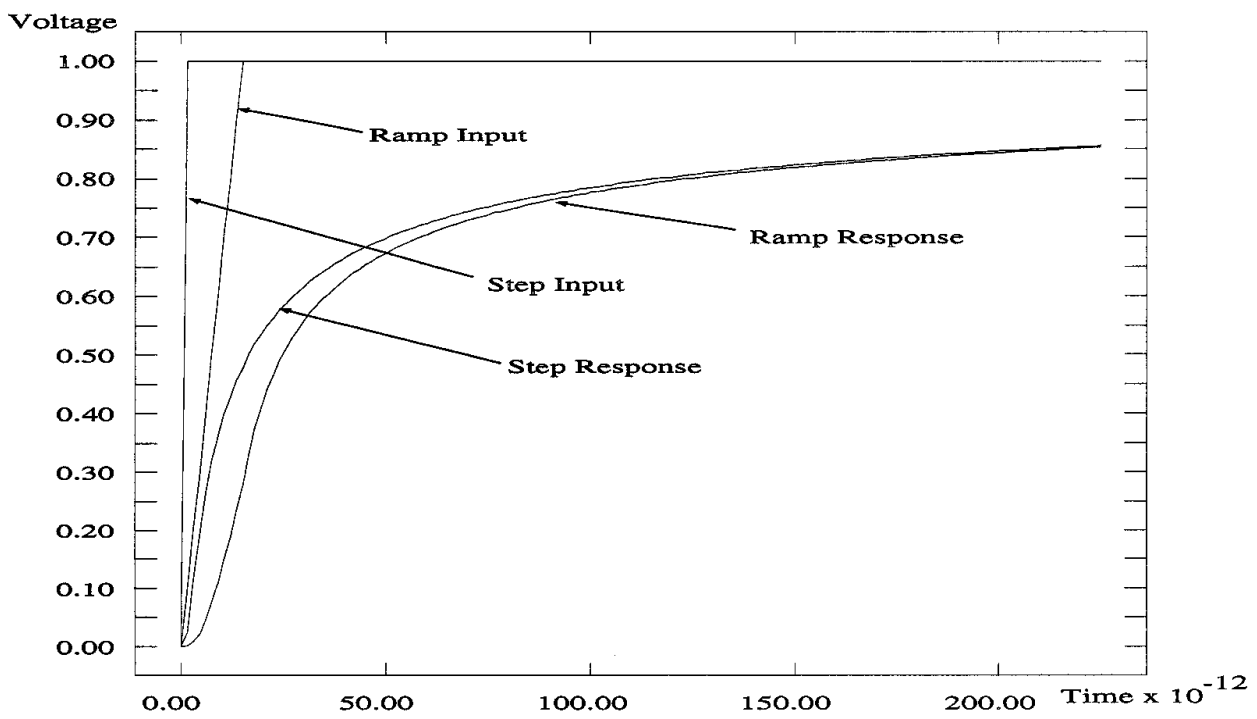

Fig. 3. Response behavior for semi-infinite line with step and finite ramp inputs. The rise-time and the position time constant $R_{x} C_{x}$ are both taken to be $15 \mathrm{ps}$.

response in the transform domain from the corresponding time-domain expression given in Eq. (4) can be obtained as

$$
V_{I}(x, s)=\frac{V_{0}}{T_{R} s^{2}}\left(1-e^{-s T_{R}}\right) e^{-\sqrt{R_{x} C_{x} s}}=V_{\text {in }}(s) e^{-b \sqrt{s}}
$$

where $V_{i n}(s)=V_{0} / T_{R} s^{2}\left(1-e^{-s T_{R}}\right)$ represents the finite ramp input shown in Figure 2(a). Figure 3 shows the substantial difference in the response for step versus ramp input. ${ }^{4}$ The above analytical expressions for the ramp input response allow direct and efficient computation of delay estimates for ramp and piecewise-linear inputs, which may enhance present-day iterative methodologies for interconnect optimization.

We may rewrite the infinite ramp response of Equation (3) in terms of a new time variable $\tau=\left(t / R_{x} C_{x}\right)$ to obtain

$$
u_{I}(x, t)=\frac{V_{0}}{\tau_{R}}\left[\left(\tau+\frac{1}{2}\right) \operatorname{erfc}\left(\sqrt{\frac{1}{4 \tau}}\right)-\sqrt{\frac{\tau}{\pi}} e^{-1 /(4 \tau)}\right] U(t)
$$

where $\tau_{R}=T_{R} / R_{x} C_{x}$ is a "relative" rise time (i.e., normalized to the time constant of the position $x$ of the line). Observe that the response at a given position on the semi-infinite line is a function of only the input rise time and the new time variable $\tau$. Thus, the relative delay (defined as threshold

\footnotetext{
${ }^{4}$ Recall that the response for the semi-infinite line under step input [Kahng and Muddu 1994] is $v_{I}(x, t)=V_{0} \operatorname{erfc}(b / \sqrt{4 t})$.
} 


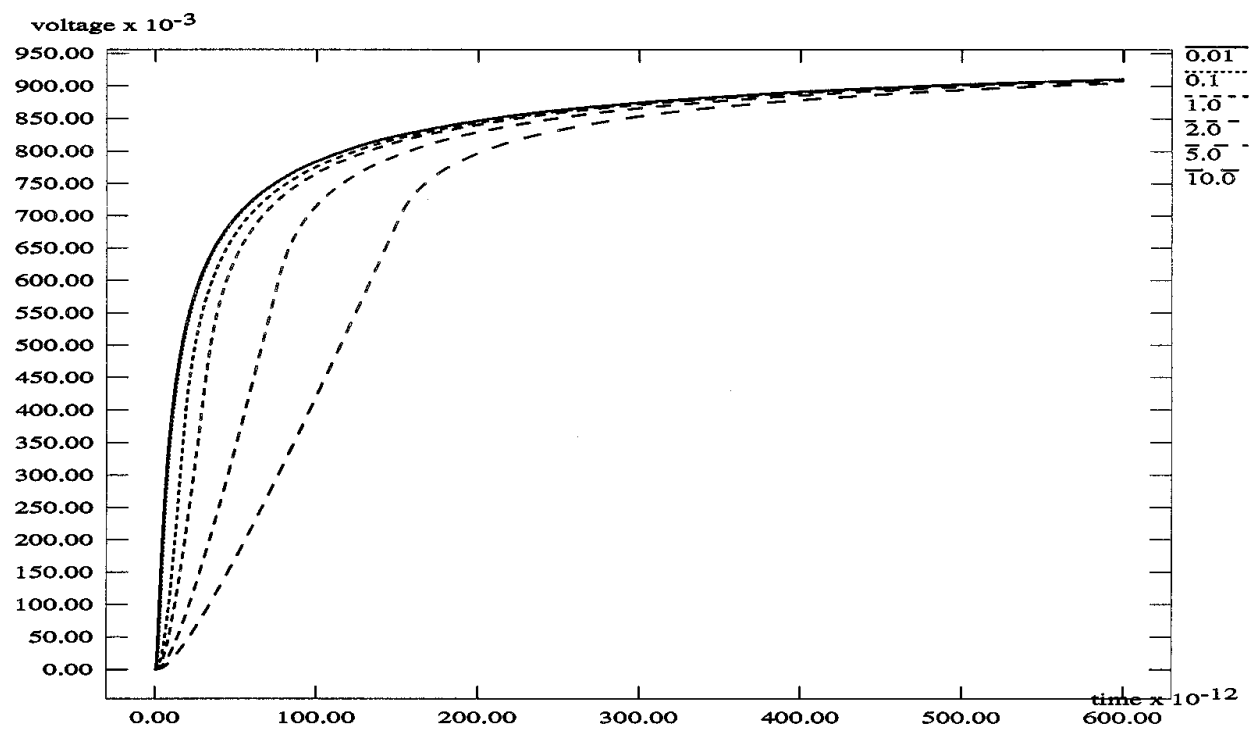

Fig. 4. Response for finite ramp input under different relative rise times $\tau_{R}=T_{R} / R_{x} C_{x}$. The position time constant $R_{x} C_{x}$ is taken to be $15 \mathrm{ps}$.

delay $D_{t h}$ divided by $R_{x} C_{x}$ ) under ramp input is a function of only $\tau_{R}$, i.e.,

$$
\frac{D_{t h}}{R_{x} C_{x}}=f\left(\tau_{R}\right)
$$

Figure 4 plots the ramp input response for various example rise times. An accurate model for interconnect delay under ramp input can be obtained by fitting a polynomial in the single variable $\tau_{R}$, but we do not discuss such methods in the present paper.

\section{FINITE $R C$ LINE ANALYSIS}

For a general finite $R C$ line with source and load impedance as shown in Figure 5, the incident propagation of voltage in the transform domain can be obtained as

$$
V_{I}(x, s)=V_{A}(s) e^{-\sqrt{R_{x} C_{x}}}
$$

The voltage at the front end of the line (i.e., at A) is

$$
V_{A}(s)=V_{i n}(s) \cdot \frac{Z_{0}}{Z_{0}+Z_{S}}=V_{i n}(s) \cdot \frac{\left(1-\Gamma_{S}(s)\right)}{2}
$$

where $\Gamma_{S}(s)$ is the reflection coefficient at the source. So, the incident 


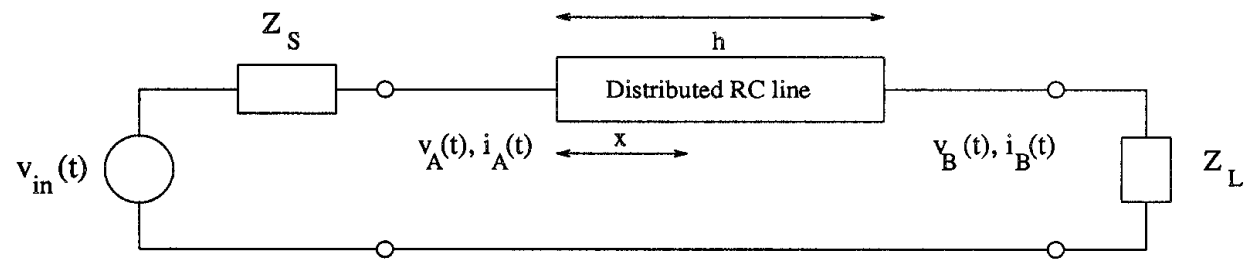

Fig. 5. A distributed $R C$ line of length $h$ and position $x$ along the line.

voltage on the line is

$$
V_{I}(x, s)=V_{i n}(s) \frac{\left(1-\Gamma_{S}(s)\right)}{2} e^{-\sqrt{R_{x} C_{x} s}}
$$

The total voltage for a finite line (Figure 5) is the summation of the incident diffusion component and reflected diffusion components that arise at the source $(S)$ and load $(L)$ discontinuities. In other words, the timedomain expansion for total voltage is

$$
v_{T o t}(x, t)=v_{I}(x, t)+\sum_{i=1}^{\infty} v_{R_{i}}(x, t)
$$

where $v_{I}(x, t) \equiv$ voltage due to the incident diffusion and $v_{R_{i}}(x, t) \equiv$ voltage due to the $i$ th reflection. (In our notation, $R_{i}$ refers to the $i$ th reflected diffusion starting from either the source or the load discontinuity; $i$ basically represents the number of trips up and down the line.) Similarly, the total voltage in the transform domain is

$$
V_{T o t}(x, s)=V_{I}(x, s)+\sum_{i=1}^{\infty} V_{R_{i}}(x, s) .
$$

In general, $v_{R_{i}}(x, t)$ can be calculated through convolution of the reflected diffusion (taking into account position displacement) with the reflection coefficients $\gamma_{S}(t)$ or $\gamma_{L}(t)$. (Note that, e.g., $\gamma_{S}(t)=\mathscr{L}^{-1}\left\{\Gamma_{S}(s)\right\}$.)

The reflection coefficient at the source in the transform domain is $\Gamma_{S}(s)=\left(Z_{S}-Z_{0}\right) /\left(Z_{S}+Z_{0}\right)$, and the reflection coefficient at the load is $\Gamma_{L}(s)=\left(Z_{L}-Z_{0}\right) /\left(Z_{L}+Z_{0}\right)$. As shown in Kahng and Muddu [1996], the voltage at the position $x$ in Figure 5 due to the first reflection at the load can be calculated from the incident wave and shifting in position by $h+$ $h-x=2 h-x$, i.e.,

$$
V_{R_{1}}(x, s)=\Gamma_{L}(s) V_{I}(2 h-x, s) .
$$


The corresponding time-domain expression is

$$
v_{R_{1}}(x, t)=\int_{\tau=0}^{t} \gamma_{L}(t-\tau) v_{I}(2 h-x, \tau) d \tau,
$$

i.e., the first reflected voltage travels distance $h$ to the end of the line before reflection, then additional distance $h-x$ to reach the specified location. Another explanation for the reflection voltages is by applying the symmetry argument in the Method of Images (or Reflections) [Kevorkian 1990; Kahng and Muddu 1996] to satisfy the boundary condition at the end of the line $x=h$. The total voltage on the line can also be proved to be equal to the sum of incident and reflected diffusion components by considering the response obtained from the 2-port transfer function of the line [Kahng and Muddu 1996]. The total voltage can be expressed in the transform domain as a summation of various reflected components

$$
\begin{aligned}
& V_{T o t}(x, s) \\
& \quad=V_{I}(x, s)+\sum_{n=1}^{\infty}\left(\Gamma_{L}^{n}(s) \Gamma_{S}^{n-1} V_{I}(2 n h-x, s)+\Gamma_{L}^{n}(s) \Gamma_{S}^{n}(s) V_{I}(2 n h+x, s)\right)
\end{aligned}
$$

and the time-domain response is

$$
\begin{aligned}
v_{T o t}(x, t) & =v_{I}(x, t)+\sum_{n=1}^{\infty}\left[a_{n}(t) \otimes v_{I}(2 n h+x, t)+b_{n}(t) \otimes v_{I}(2 n h+x, t)\right] \\
& =v_{I}(x, t)+\sum_{n=1}^{\infty}\left[\int_{\tau=0}^{t} a_{n}(t-\tau) v_{I}(2 n h-x, \tau) d \tau\right. \\
& \left.+\int_{\tau=0}^{t} b_{n}(t-\tau) v_{I}(2 n h+x, \tau) d \tau\right]
\end{aligned}
$$

where $a_{n}(t)$ and $b_{n}(t)$ represent odd and even $n$th reflection coefficient values. This methodology allows us to compute the response under ramp input for various cases of the finite-length line. The total response can be approximated by considering the analytical expressions for the first few reflection components; for higher accuracy, additional terms can be incorporated by using numerical techniques. 


\section{OPEN-ENDED FINITE RC LINE ANALYSIS}

As noted in the Introduction, the finite open-ended line with step input has been extensively treated in the literature. Such a line with ideal source has $Z_{S}=0$ (i.e., $\Gamma_{S}(s)=-1$ and $\gamma_{S}(t)=\delta(t)$ ) and $Z_{L}=\infty$ (i.e., $\Gamma_{L}(s)=1$ and $\left.\gamma_{L}(t)=\delta(t)\right)$. Proceeding from Eqs. (3) and (4), the time-domain response of incident diffusion component for the infinite ramp can be expressed in terms of the line time constant $R_{h} C_{h}$ as

$$
\begin{aligned}
u_{I}(x, t)= & \frac{V_{0}}{T_{R}}\left[\left(t+\frac{b^{2}}{2}\right) \operatorname{erfc}\left(\frac{b}{\sqrt{4 t}}\right)-b \sqrt{\frac{t}{\pi}} e^{-b^{2} /(4 t)}\right] U(t) \\
= & \frac{V_{0}}{T_{R}}\left[\left(t+\frac{x^{2}}{2 h^{2}} R_{h} C_{h}\right) \operatorname{erfc}\left(\frac{x}{h} \sqrt{\frac{R_{h} C_{h}}{4 t}}\right)\right. \\
& \left.-\frac{x}{h} \sqrt{\frac{R_{h} C_{h} t}{\pi}} e^{-(x / h)^{2} R_{h} C_{h} /(4 t)}\right] U(t)
\end{aligned}
$$

where $b=\sqrt{R_{x} C_{x}}=(x / h) \sqrt{R_{h} C_{h}}$, and $R_{h}=h r$ and $C_{h}=h c$ respectively denote the total resistance and total capacitance of the finite $R C$ line. Hence, the time-domain response of the incident diffusion component for the finite ramp is

$$
\begin{aligned}
v_{I}(x, t)= & \frac{V_{0}}{T_{R}}\left[\left(t+\frac{b^{2}}{2}\right) \operatorname{erfc}\left(\frac{b}{\sqrt{4 t}}\right)-b \sqrt{\frac{t}{\pi}} e^{-b^{2} /(4 t)}\right] U(t) \\
& -\frac{V_{0}}{T_{R}}\left[\left(t-T_{R}+\frac{b^{2}}{2}\right) \operatorname{erfc}\left(\frac{b}{\sqrt{4\left(t-T_{R}\right)}}\right)\right. \\
& \left.+b \sqrt{\frac{\left(t-T_{R}\right)}{\pi}} e^{-b^{2} /\left(4\left(t-T_{R}\right)\right)}\right] U\left(t-T_{R}\right) .
\end{aligned}
$$

Since all the coefficients of reflection are constants for an open-ended line with an ideal source, the total response can be computed from the incident diffusion component via shifts in the time variable, i.e., the total response in the transform domain is

$$
\begin{aligned}
V_{T o t}(x, s)= & V_{I}(x, s)+\sum_{n=1}^{\infty}\left[\Gamma_{L}^{n}(s) \Gamma_{S}^{n-1}(s) V_{I}(2 n h-x, s)\right. \\
& \left.+\Gamma_{L}^{n}(s) \Gamma_{S}^{n}(s) V_{I}(2 n h+x, s)\right] \\
= & V_{I}(x, s)+\sum_{n=1}^{\infty}\left[(-1)^{n-1} V_{I}(2 n h-x, s)+(-1)^{n} V_{I}(2 n h+x, s)\right]
\end{aligned}
$$




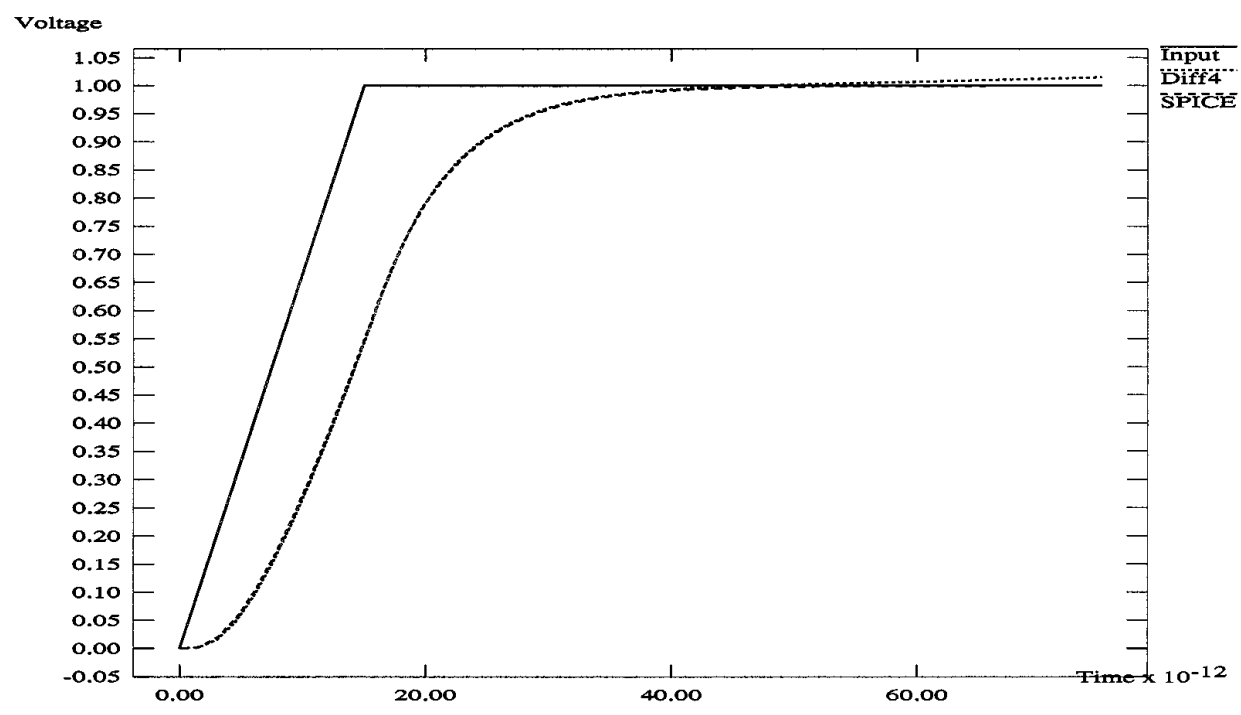

Fig. 6. Response at the end of an open-ended line for a finite ramp with rise time $T_{R}=$ $R_{h} C_{h}$, using SPICE and an approximation which sums up to the first four reflected diffusion components.

and the corresponding time-domain expression is

$$
v_{T o t}(x, t)=v_{I}(x, t)+\sum_{n=1}^{\infty}\left[(-1)^{n-1} v_{I}(2 n h-x, t)+(-1)^{n} v_{I}(2 n h+x, t)\right]
$$

where $V_{I}(x, s)$ and $v_{I}(x, t)$ represent the incident diffusion per Eqs. (5) and (8). Figure 6 compares the voltage response at the end of the line between SPICE and an approximation which sums only up to the first four reflected diffusion components.

\section{FINITE RC LINE WITH CAPACITIVE LOAD}

In this section, we derive the time-domain response under ramp input for a finite distributed $R C$ line of length $h$ with capacitive load $C_{L}$ at the end of the line (Figure 7). To our knowledge, the results of this section are also completely new. Recall from Equation (7) that the total response on the line is obtained by summing an infinite series of diffusion components due to reflections at the load and source. We will see that approximating the total response by considering up to just the first four reflections is already quite close to the SPICE-computed response. We now review the calculation of these four components (some details must be omitted for space reasons). If desired (e.g., for larger loads than those we consider), more reflection components can be calculated by using numerical techniques which, along with a general recursive equation for computing the higher order diffusion components, are discussed in Section 6. 


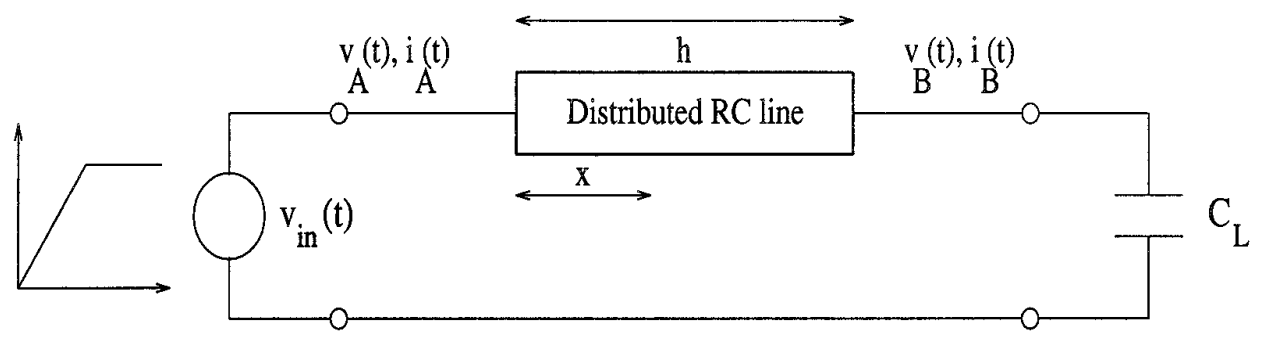

Fig. 7. Distributed $R C$ line of length $h$ with capacitive load $C_{L}$.

Incident Diffusion. The first component of the total response is the incident diffusion voltage, derived in Eqs. (3) and (4):

$$
\begin{aligned}
& u_{I}(x, t)=\frac{V_{0}}{T_{R}}\left[\left(t+\frac{b^{2}}{2}\right) \operatorname{erfc}\left(\frac{b}{\sqrt{4 t}}\right)-b \sqrt{\frac{t}{\pi}} e^{-b^{2} /(4 t)}\right] U(t) \\
& v_{I}(x, t)=\frac{V_{0}}{T_{R}}\left[\left(t+\frac{b^{2}}{2}\right) \operatorname{erfc}\left(\frac{b}{\sqrt{4 t}}\right)-b \sqrt{\frac{t}{\pi}} e^{-b^{2} /(4 t)}\right] U(t) \\
& -\frac{V_{0}}{T_{R}}\left[\left(t-T_{R}+\frac{b^{2}}{2}\right) \operatorname{erfc}\left(\frac{b}{\sqrt{4\left(t-T_{R}\right)}}\right)+b \sqrt{\frac{\left(t-T_{R}\right)}{\pi}} e^{-b^{2} /\left(4\left(t-T_{R}\right)\right)}\right] U\left(t-T_{R}\right) .
\end{aligned}
$$

Diffusion components for reflections are computed by multiplying the reflection coefficients with the incident diffusion response in the transform domain as described in Section 3.

First Reflection. The reflection coefficient at the load for a load impedance of $Z_{L}=1 /\left(s C_{L}\right)$ is

$$
\Gamma_{L}(s)=\frac{Z_{L}-Z_{0}}{Z_{L}+Z_{0}}=\frac{1-q \sqrt{s}}{1+q \sqrt{s}}
$$

where $q=C_{L} \sqrt{R_{h} / C_{h}}=\left(C_{L} / C_{h}\right) \sqrt{R_{h} C_{h}}$. The voltage response of the first reflected diffusion can be obtained from Eq. (7):

$$
\begin{aligned}
V_{R_{1}}(x, s) & =\Gamma_{L} V_{I}(2 h-x, s) \\
& =\frac{V_{0}}{T_{R} s^{2}}\left(1-e^{-s T_{R}}\right) \frac{1-q \sqrt{s}}{1+q \sqrt{s}} \exp \left(-\frac{(2 h-x)}{h} \sqrt{s R_{h} C_{h}}\right) .
\end{aligned}
$$


The infinite ramp input response is

$$
\begin{aligned}
U_{R_{1}}(x, s) & =\frac{V_{0}}{T_{R} s^{2}} \frac{1-q \sqrt{s}}{1+q \sqrt{s}} \exp \left(-\frac{(2 h-x)}{h} \sqrt{s R_{h} C_{h}}\right) \\
& =\frac{V_{0}}{T_{R} s^{2}} \frac{1-q \sqrt{s}}{1+q \sqrt{s}} e^{-b \sqrt{s}}
\end{aligned}
$$

where $b=((2 h-x) / h) \sqrt{R_{h} C_{h}}$. To compute the time-domain response we express the response in the transform domain in the form of $F(\sqrt{s}) / \sqrt{s}$. Let

$$
F_{1}(s)=\frac{V_{0}}{T_{R} s^{3}} \frac{1-q s}{1+q s} e^{-b s}=\frac{V_{0}}{T_{R}}\left[\frac{1}{s^{3}}-\frac{2 q}{s^{2}}+\frac{2 q^{2}}{s}-\frac{2 q^{2}}{(s+1 / q)}\right] e^{-b s} .
$$

The response in the transform domain can be expressed as

$$
U_{R_{1}}(x, s)=\frac{F(\sqrt{s})}{\sqrt{s}}=\frac{V_{0}}{T_{R}}\left[\frac{1}{s^{2}}-\frac{2 q}{s^{3 / 2}}+\frac{2 q^{2}}{s}-\frac{2 q^{2}}{\sqrt{s}(\sqrt{s}+1 / q)}\right] e^{-b \sqrt{s}}
$$

The inverse transform of $U_{R_{1}}(x, s)$ can be computed by inverting each term of the above equation using the identity proved in Appendix A, so that the time-domain response for the first reflected diffusion is obtained as

$$
\begin{aligned}
u_{R_{1}}(x, t)= & \frac{V_{0}}{T_{R}}\left[\left(t+\frac{b^{2}}{2}\right) \operatorname{erfc}\left(\frac{b}{\sqrt{4 t}}\right)-b \sqrt{\frac{t}{\pi}} e^{-b^{2} /(4 t)}-4 q \sqrt{\frac{t}{\pi}} e^{-b^{2} /(4 t)}\right. \\
& +2 q b \operatorname{erfc}\left(\frac{b}{\sqrt{4 t}}\right) \\
& \left.+2 q^{2} \operatorname{erfc}\left(\frac{b}{\sqrt{4 t}}\right)-2 q^{2} e^{(t+q b) / q^{2}} \operatorname{erfc}\left(\frac{\sqrt{t}}{q}+\frac{b}{2 \sqrt{t}}\right)\right] U(t) .
\end{aligned}
$$

Thus, for a finite ramp input the time-domain response is given by

$$
v_{R_{1}}(x, t)=u_{R_{1}}(x, t)-u_{R_{1}}\left(x, t-T_{R}\right)
$$

Second Reflection. The voltage response of the second reflected diffusion component due to the source discontinuity with $\Gamma_{S}(s)=\left(Z_{S}-Z_{0}\right) /$ $\left(Z_{S}+Z_{0}\right)=-1$ is given by

$$
V_{R_{2}}(x, s)=\Gamma_{S}(s) \Gamma_{L}(s) V_{I}(2 h+x, s)=-\Gamma_{L}(s) V_{I}(2 h+x, s) .
$$


The time-domain response for infinite ramp input can be calculated from the first reflected diffusion component as

$$
\begin{aligned}
u_{R_{2}}(x, t)= & \frac{V_{0}}{T_{R}}\left[-\left(t+\frac{b^{2}}{2}\right) \operatorname{erfc}\left(\frac{b}{\sqrt{4 t}}\right)+b \sqrt{\frac{t}{\pi}} e^{-b^{2} / 4 t}+4 q \sqrt{\frac{t}{\pi}} e^{-\left(b^{2} /(4 t)\right)}\right. \\
& -2 q b \operatorname{erfc}\left(\frac{b}{\sqrt{4 t}}\right) \\
& \left.-2 q^{2} \operatorname{erfc}\left(\frac{b}{\sqrt{4 t}}\right)+2 q^{2} e^{(t+q b) / q^{2}} \operatorname{erfc}\left(\frac{\sqrt{t}}{q}+\frac{b}{2 \sqrt{t}}\right)\right] U(t)
\end{aligned}
$$

where $b=((2 h+x) / h) \sqrt{R_{h} C_{h}}$, whence the time-domain response under finite ramp input is

$$
v_{R_{2}}(x, t)=u_{R_{2}}(x, t)-u_{R_{2}}\left(x, t-T_{R}\right)
$$

Third Reflection. In the transform domain, the third reflected diffusion component is

$$
\begin{aligned}
V_{R_{3}}(x, s) & =\Gamma_{S}(s) \Gamma_{L}(s)^{2} V_{I}(4 h-x, s) \\
& =\frac{-V_{0}}{T_{R} s^{2}}\left(1-e^{-s T_{R}}\right) \frac{(1-q \sqrt{s})^{2}}{(1+q \sqrt{s})^{2}} \exp \left(-\frac{(4 h-x)}{h} \sqrt{s R_{h} C_{h}}\right)
\end{aligned}
$$

and the infinite ramp response is

$$
\begin{aligned}
U_{R_{3}}(x, s) & =\frac{-V_{0}}{T_{R} s^{2}} \frac{(1-q \sqrt{s})^{2}}{(1+q \sqrt{s})^{2}} \exp \left(-\frac{(4 h-x)}{h} \sqrt{s R_{h} C_{h}}\right) \\
& =\frac{-V_{0}}{T_{R} s^{2}} \frac{(1-q \sqrt{s})^{2}}{(1+q \sqrt{s})^{2}} e^{-b \sqrt{s}} \\
& =\frac{F_{3}(\sqrt{s})}{\sqrt{s}}
\end{aligned}
$$

where $b=((4 h-x) / h) \sqrt{R_{h} C_{h}}$ and the function $F_{3}(s)$ is given by

$$
\begin{aligned}
F_{3}(s) & =-\frac{V_{0}}{T_{R} s^{3}}\left(\frac{1-q s}{1+q s}\right)^{2} e^{-b s} \\
& =\frac{V_{0}}{T_{R}}\left[-\frac{1}{s^{3}}+\frac{4 q}{s^{2}}-\frac{8 q^{2}}{s}+\frac{8 q^{2}}{(s+1 / q)}+\frac{4 q}{(s+1 / q)^{2}}\right] e^{-b s} .
\end{aligned}
$$

ACM Transactions on Design Automation of Electronic Systems, Vol. 2, No. 2, April 1997. 
Thus, the infinite ramp response can be expressed as

$$
\begin{aligned}
U_{R_{3}}(x, s) & =\frac{F_{3}(\sqrt{s})}{\sqrt{s}} \\
& =\frac{V_{0}}{T_{R}}\left[-\frac{1}{s^{2}}+\frac{4 q}{s^{3 / 2}}-\frac{8 q^{2}}{s}+\frac{8 q^{2}}{\sqrt{s}(\sqrt{s}+1 / q)}+\frac{4 q}{\sqrt{s}(\sqrt{s}+1 / q)^{2}}\right] e^{-b \sqrt{s}}
\end{aligned}
$$

The inverse transform of $U_{R_{3}}(x, s)$ can be computed by inverting each term of the above equation; the inverse transform of the first four terms can be obtained from the analysis of the First Reflection, above. The time-domain expression for the last term can be calculated by considering the function

$$
F_{4}(s)=\frac{4 q}{(s+1 / q)^{2}} e^{-b s}
$$

whose corresponding time-domain function is $f_{4}(t)=4 q(t-b) e^{-(t-b) / q}$ $U(t-b)$. The inverse transform of the last term is obtained using the identity in Appendix A as

$$
\begin{aligned}
f_{4}(x, t)= & \frac{1}{\sqrt{\pi t}} \int_{x=0}^{\infty} e^{-x^{2} /(4 t)} f_{4}(x) d x \\
= & \frac{4 q}{\sqrt{\pi t}} \int_{x=0}^{\infty} e^{-x^{2} /(4 t)}(x-b) e^{-(x-b) / q} d x \\
= & 8 q \sqrt{\frac{t}{\pi}} \exp \left(-\left(\frac{\sqrt{t}}{q}+\frac{b}{2 \sqrt{t}}\right)^{2}+\frac{(t+q b)}{q^{2}}\right) \\
& -4 q\left(\frac{2 t}{q}+b\right) e^{(t+q b) / q^{2}} \operatorname{erfc}\left(\frac{\sqrt{t}}{q}+\frac{b}{2 \sqrt{t}}\right) .
\end{aligned}
$$

The time-domain response for the third reflected diffusion for an infinite ramp is

$$
\begin{aligned}
u_{R_{3}}(x, t)= & \frac{V_{0}}{T_{R}}\left[-\left(t+\frac{b^{2}}{2}\right) \operatorname{erfc}\left(\frac{b}{\sqrt{4 t}}\right)+b \sqrt{\frac{t}{\pi}} e^{-b^{2} /(4 t)}+8 q \sqrt{\frac{t}{\pi}} e^{-b^{2} /(4 t)}\right. \\
& -4 q b \operatorname{erfc}\left(\frac{b}{\sqrt{4 t}}\right)
\end{aligned}
$$




$$
\begin{aligned}
& -8 q^{2} \operatorname{erfc}\left(\frac{b}{\sqrt{4 t}}\right)+8 q^{2} e^{(t+q b) / q^{2}} \operatorname{erfc}\left(\frac{\sqrt{t}}{q}+\frac{b}{2 \sqrt{t}}\right) \\
& +8 \sqrt{\frac{t}{\pi}} \exp \left(-\left(\frac{\sqrt{t}}{q}+\frac{b}{2 \sqrt{t}}\right)^{2}+\frac{(t+q b)}{q^{2}}\right) \\
& -4(2 t+q b) e^{(t+q b) / q^{2}} \operatorname{erfc}\left(\frac{\sqrt{t}}{q}+\frac{b}{2 \sqrt{t}}\right) U(t)
\end{aligned}
$$

and the finite ramp time-domain response is $v_{R_{3}}(x, t)=u_{R_{3}}(x, t)-$ $u_{R_{3}}\left(x, t-T_{R}\right)$.

Fourth Reflection. Similarly, the voltage response of the fourth reflection at the source is $V_{R_{4}}(x, s)=\Gamma_{S}(s)^{2} \Gamma_{L}(s)^{2} V_{I}(4 h+x, s)=\Gamma_{L}(s)^{2} V_{I}(4 h+x, s)$, and the time-domain response for infinite ramp input is

$$
\begin{aligned}
& u_{R_{4}}(x, t)=\frac{V_{0}}{T_{R}}\left[\left(t+\frac{b^{2}}{2}\right) \operatorname{erfc}\left(\frac{b}{\sqrt{4 t}}\right)-b \sqrt{\frac{t}{\pi}} e^{-b^{2} /(4 t)}-8 q \sqrt{\frac{t}{\pi}} e^{-b^{2} /(4 t)}\right. \\
& +4 q b \operatorname{erfc}\left(\frac{b}{\sqrt{4 t}}\right)+8 q^{2} \operatorname{erfc}\left(\frac{b}{\sqrt{4 t}}\right)-8 q^{2} e^{(t+q b) / q^{2}} \operatorname{erfc}\left(\frac{\sqrt{t}}{q}+\frac{b}{2 \sqrt{t}}\right) \\
& -8 \sqrt{\frac{t}{\pi}} \exp \left(-\left(\frac{\sqrt{t}}{q}+\frac{b}{2 \sqrt{t}}\right)^{2}+\frac{(t+q b)}{q^{2}}\right) \\
& +4(2 t+q b) e^{(t+q b) / q^{2}} \operatorname{erfc}\left(\frac{\sqrt{t}}{q}+\frac{b}{2 \sqrt{t}}\right) U(t)
\end{aligned}
$$

where $b=((4 h+x)) / \sqrt{R_{h} C_{h}}$; the response for the finite ramp input is $v_{R_{4}}(x, t)=u_{R_{4}}(x, t)-u_{R_{4}}\left(x, t-T_{R}\right)$. If we approximate the total response for infinite ramp input by considering only up to these first four reflections, we have

$$
u_{T o t}(x, t) \approx u_{I}(x, t)+u_{R_{1}}(x, t)+u_{R_{2}}(x, t)+u_{R_{3}}(x, t)+u_{R_{4}}(x, t)
$$

and the total response for the finite ramp input is

$$
\begin{aligned}
v_{T o t}(x, t) & =u_{t o t}(x, t)-u_{T o t}\left(x, t-T_{R}\right) \\
& \approx\left[v_{I}(x, t)+v_{R_{1}}(x, t)+v_{R_{2}}(x, t)+v_{R_{3}}(x, t)+v_{R_{4}}(x, t)\right] .
\end{aligned}
$$

We call the approximation of Eq. (14) the Diff4 model. Table I compares Diff4 delay estimates at different threshold values for a wide range of capacitive loads, versus the SPICE URC (Uniform Distributed $R C$ ) model. 
Table I.

A comparison of delay values at the end of the line $(x=h)$ between SPICE URC model and the analytical expression computed from diffusion analysis using up to the first four reflection components. The input rise time considered to be equal to line time constant, $T_{R}=R_{h} C_{h}=15$ psec.

\begin{tabular}{|c||c|c||c|c||}
\hline \multicolumn{1}{|c||}{$\begin{array}{c}\text { Load factor } \\
\left(\frac{C_{L}}{C_{h}}\right)\end{array}$} & 10\% Threshold delays $\left(R_{h} C_{h}\right)$ & \multicolumn{2}{c||}{ 50\% Threshold delays $\left(R_{h} C_{h}\right)$} \\
\cline { 2 - 5 } & SPICE & Diff4 & SPICE & Diff4 \\
\hline \hline 0.0 & 6.20 & 6.30 & 14.20 & 14.25 \\
0.25 & 7.60 & 7.70 & 16.90 & 16.85 \\
0.5 & 8.70 & 8.80 & 19.35 & 19.30 \\
1.0 & 10.50 & 10.50 & 24.30 & 24.25 \\
2.0 & 13.10 & 13.15 & 34.20 & 33.50 \\
5.0 & 18.50 & 17.55 & 64.00 & 56.00 \\
\hline \hline Load factor & $\mathbf{6 3 . 2 \%}$ Threshold delays $\left(R_{h} C_{h}\right)$ & 90\% Threshold delays $\left(R_{h} C_{h}\right)$ \\
\cline { 2 - 5 }$\left(\frac{C_{L}}{C_{h}}\right)$ & SPICE & Diff4 & SPICE & Diff4 \\
\hline \hline 0.0 & 16.50 & 16.50 & 24.75 & 24.70 \\
0.25 & 19.75 & 19.70 & 32.00 & 30.00 \\
0.5 & 23.30 & 23.30 & 40.00 & 38.90 \\
1.0 & 30.50 & 30.60 & 57.50 & 57.75 \\
2.0 & 45.10 & 44.70 & 92.00 & 99.00 \\
5.0 & 89.50 & 80.00 & 199.00 & 235.00 \\
\hline
\end{tabular}

The delay estimates using our new diffusion equation approach are very close to the SPICE-computed delays, even though only four reflections are considered. Figure 8 gives a comparison of the voltage response between SPICE and the Diff4 model for the case of load factor $C_{L} / C_{h}=1$.0. Since the response is computed solely by evaluating of diffusion component expressions, the efficiency of this approach is at least 2 or 3 orders of magnitude faster than SPICE.

\section{GENERALIZATION OF THE REFLECTED COMPONENT COMPUTATION}

While the previous section gave analytical expressions for the first four reflection components, we now discuss methods to compute higher-order components of the infinite ramp response; from these, the components of the finite ramp response easily follow. In general, the (infinite or finite) ramp response in the transform domain is a function of reflection coefficients and the incident voltage. From Eq. (6), the $2 n$th reflected component at the source is given by $U_{R_{2 n}}(x, s)=\Gamma_{S}(s)^{n} \Gamma_{L}(s)^{n} U_{I}(2 n h+x, s)$, and the $(2 n-1)$ th reflected component at the load is similarly given by $U_{R_{2 n-1}}(x, s)$ $=\Gamma_{S}(s)^{n-1} \Gamma_{L}(s)^{n} U_{I}(2 n h-x, s)$. The time-domain response for each reflected diffusion component can be computed using the above method (Section 5) for obtaining an exact analytical expression. To generalize the computation of each time-domain reflected diffusion component, we may apply two different techniques: (i) a numerical integration approach, and (ii) recursive error function evaluation. 


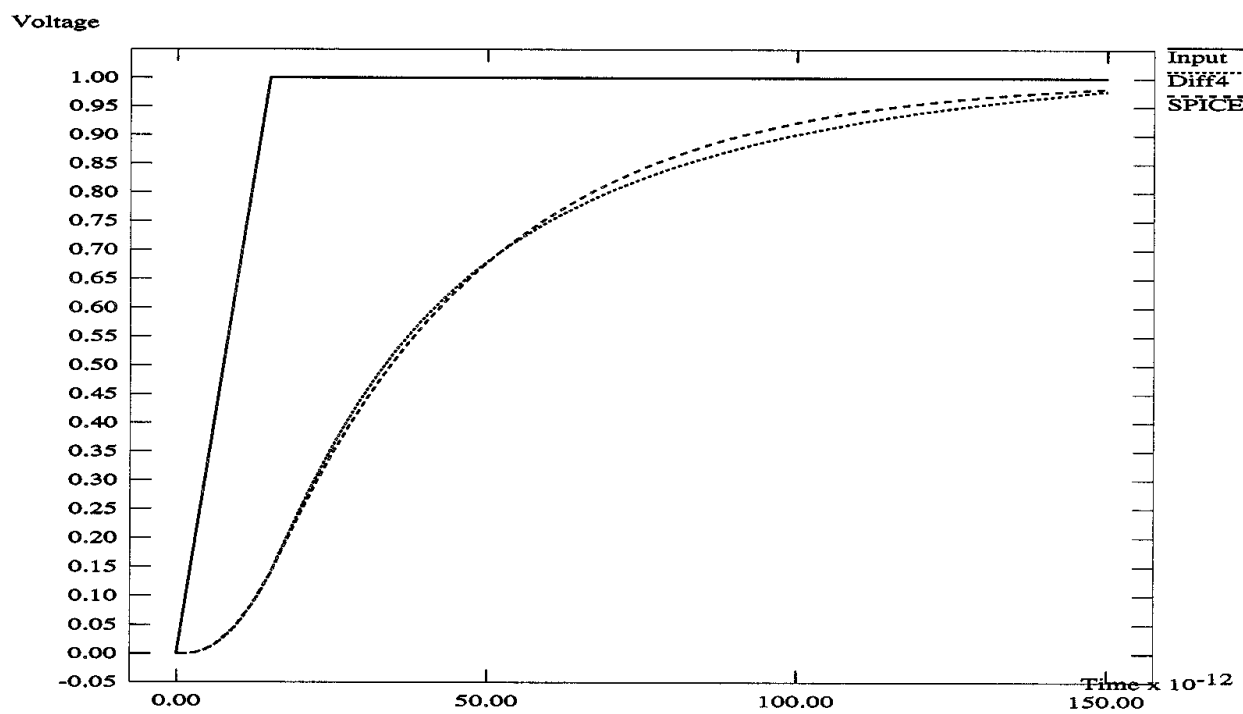

Fig. 8. Response for a finite $R C$ line with capacitive load under finite ramp input, calculated using both the SPICE URC model and the Diff4 model. The rise time of the input is $T_{R}=$ $R_{h} C_{h}$ and the load factor is $C_{L} / C_{h}=1.0$.

\subsection{A Numerical Integration Approach}

Here and in the next section, we consider the time-domain expression for the $2 n$th source reflection component (the $(2 n-1)$ th load reflection component is analogous). Assuming resistive source and capacitive load impedances, the reflection coefficients can be represented as

$$
\Gamma_{S}(s)=-\frac{(1-p \sqrt{s})}{(1+p \sqrt{s})} \quad \Gamma_{L}(s)=\frac{(1-q \sqrt{s})}{(1+q \sqrt{s})}
$$

where $p=R_{S} / R_{h} \sqrt{R_{h} C_{h}}$ and $q=C_{L} / C_{h} \sqrt{R_{h} C_{h}}$. Substituting for the reflection coefficients and incident voltage and using $b=((2 n h+x) / h) \sqrt{R_{h} C_{h}}$, the infinite ramp response in the transform domain is given by

$$
\begin{aligned}
U_{R_{2 n}}(x, s) & =\Gamma_{L}^{n}(s) \Gamma_{S}^{n}(s) V_{I}(2 n h+x, s)=\Gamma_{L}^{n}(s) \Gamma_{S}^{n}(s) \frac{V_{0} e^{-b \sqrt{s}}}{T_{R} s^{2}} \\
& =(-1)^{n} \frac{V_{0}}{T_{R} s^{2}} \frac{(1-p \sqrt{s})^{n}}{(1+p \sqrt{s})^{n+1}} \frac{(1-q \sqrt{s})^{n}}{(1+q \sqrt{s})^{n}} e^{-b \sqrt{s}} .
\end{aligned}
$$

The response in the time domain for this reflected component can be evaluated by expressing $U_{R_{2 n}}$ in the form $F(\sqrt{s}) / \sqrt{s}$ and applying the identity of Appendix A. To evaluate the integral in the identity we need the time-domain expression $f(t)$ of the function. Hence, we first apply partial 
fraction expansion to the function as

$$
\begin{aligned}
F(s)= & (-1)^{n} \frac{V_{0}}{T_{R} s^{3}} \frac{(1-p s)^{n}}{(1+p s)^{n+1}} \frac{(1-q s)^{n}}{(1+q s)^{n}} e^{-b s} \\
= & \frac{V_{0}(-1)^{n}}{p T_{R}}\left[\frac{D_{1}}{s}+\frac{D_{2}}{s^{2}}+\frac{D_{3}}{s^{3}}+\frac{A_{1}}{(s+1 / p)}+\frac{A_{2}}{(s+1 / p)^{2}}+\ldots\right. \\
& \left.+\frac{A_{n+1}}{(s+1 / p)^{n+1}}+\frac{B_{1}}{(s+1 / q)}+\frac{B_{2}}{(s+1 / q)^{2}}+\ldots+\frac{B_{n}}{s+1 / q)^{n}}\right] e^{-b s}
\end{aligned}
$$

where $A_{i}, B_{i}$, and $D_{i}$ are the coefficients corresponding to each pole of the function. The inverse transform for this function is easily obtained as

$$
\begin{aligned}
f(t)= & \frac{V_{0}(-1)^{n}}{p T_{R}}\left[D_{1}+D_{2}(t-b)+D_{3} \frac{(t-b)^{2}}{2}+A_{1} e^{(t-b) / p}\right. \\
& +\ldots+\frac{A_{n+1}}{n !}(t-b)^{n} e^{(t-b) / p}+B_{1} e^{(t-b) / q}+\ldots \\
& \left.+\frac{B_{n}}{(n-1) !}(t-b)^{n-1} e^{(t-b) / q}\right] U(t-b)
\end{aligned}
$$

and the time-domain response for the reflection component $U_{R_{2 n}}(x, s)$ can be calculated by numerical integration as

$$
u_{R_{2 n}}(x, t)=\frac{1}{\sqrt{\pi t}} \int_{x=0}^{\infty} e^{-x^{2} /(4 t)} f(x) d x
$$

\subsection{Recursive Error Function Evaluation}

A final (an alternative) for calculating the time-domain response is by recursive error function evaluation. Instead of calculating the inverse transform of the function $F(s)$ and using numerical integration, we may rewrite the reflected component in the transform domain in the form $F(\sqrt{s}) / \sqrt{s}$ and then calculate the inverse transform, i.e.,

$$
\begin{aligned}
U_{R_{2 n}}(x, s)= & \frac{F(\sqrt{s})}{\sqrt{s}} \\
= & \frac{V_{0}(-1)^{n}}{p T_{R}}\left[\frac{D_{1}}{s}+\frac{D_{2}}{s^{3 / 2}}+\frac{D_{3}}{s^{2}}+\frac{A_{1}}{\sqrt{s}(\sqrt{s}+1 / p)}+\frac{A_{2}}{\sqrt{s}(\sqrt{s}+1 / p)^{2}}\right. \\
& +\ldots+\frac{A_{n+1}}{\sqrt{s}(\sqrt{s}+1 / p)^{n+1}}+\frac{B_{1}}{\sqrt{s}(\sqrt{s}+1 / q)}+\frac{B_{2}}{\sqrt{s}(\sqrt{s}+1 / q)^{2}}
\end{aligned}
$$




$$
\left.+\ldots+\frac{B_{n}}{\sqrt{s}(\sqrt{s}+1 / q)^{n}}\right] e^{-b \sqrt{s}}
$$

The inverse transform for $U_{R_{2 n}}(x, s)$ can now be obtained by taking inverse transforms separately for each term in the above expression. The timedomain expression for the $2 n$th reflection component can be obtained in the form of recursive error functions as (see Appendix B) ${ }^{5}$

$$
\begin{aligned}
u_{R_{2 n}}(x, t)= & \frac{V_{0}(-1)^{n}}{p T_{R}}\left[D_{1} e r f c\left(\frac{b}{\sqrt{4 t}}\right)+2 D_{2} \sqrt{\frac{t}{\pi}} e^{-b^{2} /(4 t)}-b D_{2} e r f c\left(\frac{b}{\sqrt{4 t}}\right)\right. \\
& +D_{3}\left(t+\frac{b^{2}}{2}\right) \operatorname{erfc}\left(\frac{b}{\sqrt{4 t}}\right)-b D_{3} \sqrt{\frac{t}{\pi}} e^{-b^{2} /(4 t)} \\
& +\sum_{k=1}^{n+1} A_{k}(4 t)^{((n-1) / 2)} \exp \left(\frac{t}{p^{2}}-\frac{b}{p}\right) \operatorname{erfc}_{(n-1)}\left(\frac{b-(2 t / p)}{\sqrt{4 t}}\right) \\
& +\sum_{k=1}^{n} B_{k}(4 t)^{((n-1) / 2)} \exp \left(\frac{t}{q^{2}}-\frac{b}{q}\right) \operatorname{erfc}_{(n-1)}\left(\frac{b-(2 t / q)}{\sqrt{4 t}}\right)
\end{aligned}
$$

which can be evaluated using the recursive expression for the error function [Abramowitz and Stegun 1972], i.e.,

$$
\begin{aligned}
\operatorname{erfc}_{(n)}(z) & =-\frac{z}{n} \operatorname{erfc}(n-1) \\
& (z)+\frac{1}{2 n} \operatorname{erfc_{(n-2)}}(z) \\
& =\frac{2}{\sqrt{\pi}} \int_{t=z}^{\infty} \frac{(t-z)^{n}}{n !} e^{-t^{2}} d t
\end{aligned}
$$

Thus, the time-domain response for the finite ramp input can be obtained as

$$
v_{R_{2 n}}(x, t)=u_{R_{2 n}}(x, t)-u_{R_{2 n}}\left(x, t-T_{R}\right)
$$

\section{CONCLUSIONS}

We have analyzed finite distributed $R C$ lines under ramp input via a new technique based on solving the diffusion equation and using reflected

\footnotetext{
${ }^{5}$ Appendix B gives two general transform pair relationships that can be used to invert the terms of general reflection component expressions. The time-domain expression of either transform pair can be written in a compact form consisting of recursive error functions, or directly as an integral form obtained from the identity in Appendix A.
} 
diffusion components to account for reflections at the source and load end of the line. Our general and, in the limit, exact approach computes the time-domain response for finite $R C$ lines under ramp input by summing distinct diffusions starting at either end of the line. We then derived the time-domain voltage response for various configurations of the $R C$ line. To the best of our knowledge, these results are completely new; there is no previous literature on this subject. Delay estimates using our new approach (the Diff4 model incorporating up to the first four reflection components) are very close to SPICE-computed (URC model) delays. Finally, we present two methodologies, including a general recursive equation, for computing the higher-order diffusion components due to reflections at either the source or load end. Ongoing work extends this approach to response computations in arbitrary interconnection trees by modeling both reflection and transmission coefficients at discontinuities, e.g., we might derive the input ramp for each interconnect from the response at the end of the previous (upstream) interconnect.

\section{APPENDIX A: LAPLACE TRANSFORM IDENTITY}

We present the following identity, which is useful in calculating timedomain expressions for the distributed $R C$ line from the corresponding transform domain expressions.

THEOREM. Let $f(t)$ and $F(s)$ be a Laplace transform pair. Then

$$
\frac{F(\sqrt{s})}{\sqrt{s}} \Leftrightarrow \frac{1}{\sqrt{\pi t}} \int_{u=0}^{\infty} e^{-u^{2} /(4 t)} f(u) d u
$$

Proof. Any function $f(u)$ can be expressed using the delta function as

$$
f(u)=\int_{u_{0}=0}^{\infty} f(u) \delta\left(u-u_{0}\right) d u_{0}=\int_{u_{0}=0}^{\infty} f\left(u_{0}\right) \delta\left(u-u_{0}\right) d u_{0} .
$$

Applying this to the time-domain function that is to be inverted, we get

$$
\begin{aligned}
\frac{1}{\sqrt{\pi t}} \int_{u=0}^{\infty} e^{-u^{2} /(4 t)} f(u) d u & =\frac{1}{\sqrt{\pi t}} \int_{u=0}^{\infty} e^{-u^{2} /(4 t)} d u\left(\int_{u_{0}=0}^{\infty} f\left(u_{0}\right) \delta\left(u-u_{0}\right) d u_{0}\right) \\
& =\frac{1}{\sqrt{\pi t}} \int_{u_{0}=0}^{\infty} f\left(u_{0}\right) d u_{0} \int_{u=0}^{\infty} e^{-u^{2} /(4 t)} \delta\left(u-u_{0}\right) d u \\
& =\frac{1}{\sqrt{\pi t}} \int_{u_{0}=0}^{\infty} f\left(u_{0}\right) e^{-u_{0}^{2} /(4 t)} d u_{0} .
\end{aligned}
$$


Taking the Laplace transform for the above equation,

$$
\begin{aligned}
\int_{t=0}^{\infty} e^{-s t} d t\left(\frac{1}{\sqrt{\pi t}} \int_{u=0}^{\infty} e^{-u^{2} /(4 t)} f(u) d u\right) & =\int_{t=0}^{\infty} e^{-s t} d t \frac{1}{\sqrt{\pi t}} \int_{u_{0}=0}^{\infty} f\left(u_{0}\right) e^{-u_{0}^{2} /(4 t)} d u_{0} \\
& =\int_{u_{0}=0}^{\infty} f\left(u_{0}\right) d u_{0} \int_{t=0}^{\infty} \frac{1}{\sqrt{\pi t}} e^{-u_{0}^{2} /(4 t)} e^{-s t} d t
\end{aligned}
$$

Note [Healey 1967] that the integral with respect to $t$ is a Laplace transform of

$$
\int_{t=0}^{\infty} \frac{1}{\sqrt{\pi t}} e^{-u_{0}^{2} /(4 t)} e^{-s t} d t=\frac{e^{-u_{0} \sqrt{s}}}{\sqrt{s}} .
$$

Substituting in the above equation and using the definition of Laplace transform, we get

$$
\begin{aligned}
\int_{t=0}^{\infty} e^{-s t} d t\left(\frac{1}{\sqrt{\pi t}} \int_{u=0}^{\infty} e^{-u^{2} /(4 t)} f(u) d u\right) & =\int_{u_{0}=0}^{\infty} f\left(u_{0}\right) \frac{e^{-u_{0} \sqrt{s}}}{\sqrt{s}} d u_{0} \\
& =\frac{1}{\sqrt{s}} \int_{u_{0}=0}^{\infty} e^{-u_{0} \sqrt{s}} f\left(u_{0}\right) d u_{0}=\frac{F(\sqrt{s})}{\sqrt{s}} .
\end{aligned}
$$

Therefore, the transform pair is given by

$$
\frac{1}{\sqrt{\pi t}} \int_{u=0}^{\infty} e^{-u^{2} /(4 t)} f(u) d u \Leftrightarrow \frac{F(\sqrt{s})}{\sqrt{s}} .
$$

\section{APPENDIX B: TRANSFORM PAIR RELATIONS FOR FUNCTIONS OF $\sqrt{s}$}

Here we present general transform pairs for functions of $\sqrt{s}$ with repeated poles, which are used in computing the time-domain diffusion response components for distributed $R C$ lines. Using these transform pairs, the diffusion components can be expressed in a compact form consisting of recursive error functions. Because of the recursive nature of the expressions, they can be easily used within a simulation methodology to compute the response as a function of time.

Transform Pair 1

$$
\frac{e^{-b \sqrt{s}}}{s^{n}} \Leftrightarrow(4 t)^{n-1} \operatorname{erfc} c_{(2 n-2)}\left(\frac{b}{\sqrt{4 t}}\right) .
$$


Proof. Consider the following function $F(s)=e^{-b s} / s^{2 n-1}$, and the function in the time-domain is given by

$$
f(t)=\frac{(t-b)^{2 n-2}}{(2 n-2) !} U(t-b)
$$

where $U(t)$ is a step function. Now from the identity in the Appendix A, we can express the required transform pair in terms of the function as

$$
\frac{e^{-b \sqrt{s}}}{s^{n}}=\frac{F(\sqrt{s})}{\sqrt{s}} .
$$

Therefore, the inverse transform is obtained as

$$
\begin{aligned}
\frac{e^{-b \sqrt{s}}}{s^{n}} & \Leftrightarrow \frac{1}{\sqrt{\pi t}} \int_{u=0}^{\infty} e^{-u^{2} /(4 t)} f(u) d u \\
& \Leftrightarrow \frac{1}{\sqrt{\pi t}} \int_{u=b}^{\infty} e^{-u^{2} /(4 t)} \frac{(u-b)^{2 n-2}}{(2 n-2) !} d u \\
& \Leftrightarrow(4 t)^{n-1} \frac{2}{\sqrt{\pi}} \int_{x=(b / \sqrt{4 t})}^{\infty} e^{-x^{2}} \frac{(x-b / \sqrt{4 t})^{2 n-2}}{(2 n-2) !} d x \\
& \Leftrightarrow(4 t)^{n-1} \operatorname{erfc} c_{(2 n-2)}\left(\frac{b}{\sqrt{4 t}}\right) .
\end{aligned}
$$

We can express the last integral in the form of $n$th error function by using the integral definition [Abramowitz and Stegun 1972],

$$
\operatorname{erfc}_{(n)}(z)=\frac{2}{\sqrt{\pi}} \int_{t=z}^{\infty} \frac{(t-z)^{n}}{n !} e^{-t^{2}} d t
$$

where $n=1,2,3, \ldots$ The $n$th error function can also be expressed recursively as

$$
\operatorname{erfc}_{(n)}(z)=-\frac{z}{n} \operatorname{erfc}_{(n-1)}(z)+\frac{1}{2 n} \operatorname{erfc}_{(n-2)}(z)
$$

with the initial cases given by

$$
\operatorname{erfc}_{(-1)}(z)=\frac{2}{\sqrt{\pi}} e^{-z^{2}} \operatorname{erfc}_{(0)}(z)=\operatorname{erfc}(z)=\frac{2}{\sqrt{\pi}} \int_{t=0}^{z} e^{-t^{2}} d t
$$


Transform Pair 2. Using the Transform Pair 1 approach, we can also obtain the following relationship:

$$
\frac{e^{-b \sqrt{s}}}{\sqrt{s}(\sqrt{s}-a)^{n}} \Leftrightarrow(4 t)^{(n-1) / 2} e^{\left(a^{2} t+a b\right)} e r f c_{(n-1)}\left(\frac{b+2 t a}{\sqrt{4 t}}\right) .
$$

\section{REFERENCES}

Abramowitz, H. M. And Stegun, I. A. 1972. Handbook of Mathematical Functions With Formulas, Graphs, and Mathematical Tables. National Bureau of Standards, Applied Mathematics Series 55.

AвUELMA'AtTI, M. T. 1989. Multipole approximation of capacitively loaded VLSI interconnections. IEEE Proceedings G 136 (June), 118-120.

Abuelma'AtTi, M. T. 1990. The waveform degradation in VLSI interconnections. IEEE J. Solid-State Circuits 25 (Aug.), 1014-1016.

Abuelma'AtTi, M. T. 1991. Modeling of capacitively (resistively)-loaded uniform RC lines for computer aided-analysis. IEEE Proceedings-G 138, 463-466.

Antinone, R. J. AND Brown, G. W. 1983. The modeling of resistive interconnects for integrated circuits. IEEE J. Solid-State Circuits 18 (April), 200-203.

BAKogLu, H. B. 1990. Circuits, Interconnections and Packaging for VLSI. Addison-Wesley.

CAmpbell, G. A. And Foster, R. M. 1957. Fourier Integrals for Practical Applications, Princeton, Van Nostrand.

ElmoRE, W. C. 1948. The transient response of damped linear networks with particular regard to wideband amplifiers. J. Appl. Phys. 19 (Jan.), 55-63.

Healey, M. 1967. Tables of Laplace, Heaviside, Fourier, and Z Transforms. W \& R Chambers Ltd.

HuANG, C. C. AND Wu, L. L. 1987. Signal degradation through module pins in VLSI packaging. IBM J. Res. Dev. 31, 4 (July), 489-498.

KAHNG, A. B. AND MudDU, S. 1994. Delay analysis of VLSI interconnections using the diffusion equation model. In Proceedings ACM/IEEE Design Automation Conference (June), 563-569.

KAHNG, A. B. AND Muddu, S. 1996. New diffusion-based analyses of distributed $R C$ interconnections. IEEE International Symposium on Circuits and Systems (May). Also available as Res. Rep. UCLA CS Dept. TR-950019.

KaHnG, A. B. AND RoBINS, G. 1994. On Optimal Interconnections for VLSI. Kluwer Academic.

Kaufman, W. M. and Garrett, S. J. 1962. Tapered distributed filters. IRE Trans. Circuit Theor. (Dec.), 329-336.

KAUPP, H. R. 1989. Waveform degradation in VLSI interconnections. IEEE J. Solid-State Circuits (Aug.), 1150-1153.

Kevorkian, J. 1990. Partial Differential Equations: Analytical Solution Techniques. Wadsworth \& Brooks/Cole.

Mattes, H. L. 1993. Behavior of a single transmission line stimulated with a step function. Manuscript, Aug.

Menezes, N., Pullela, S., Dartu, F., And Pillage, L. T. 1994. $R C$ interconnect synthesis-a moment fitting approach. In Proceedings IEEE / ACM International Conference Computer-Aided Design. (Nov.), 418-425.

Peirson, R. C. AND Bertnolli, E. C. 1969. Time-domain analysis and measurement techniques for distributed RC structures. II. Impulse measurement techniques. J. Appl. Phys. (June), $118-122$.

RAO, V. B. 1995. Delay analysis of the distributed $R C$ line. In Proceedings ACM/IEEE Design Automation Conference (June), 370-375.

SAKURAI, T. 1983. Approximation of wiring delay in MOSFET LSI. IEEE J. Solid-State Circuits SC-18 (Aug.), 418-426.

WiLnaI, A. 1971. Open-ended RC line model predicts MOSFET IC response. EDN (Dec. 15). pp. $53-54$.

Received December 1995; revised August 1996; accepted September 1996 